\title{
Effect of different charges of modified electroconvulsive seizure on the cognitive behavior in stressed rats: Effects of GluR1 phosphorylation and CaMKII $\alpha$ activity
}

\author{
FAN ZHANG ${ }^{1}$, GUIHUA HUANG ${ }^{2}$ and XIANLIN ZHU ${ }^{3}$ \\ ${ }^{1}$ Department of Anesthesiology, The People's Hospital of Jianyang City, Chengdu, Sichuan 610000; \\ ${ }^{2}$ Department of Anesthesiology, The First People's Hospital of Zunyi, Zunyi, Guizhou 563000; ${ }^{3}$ Department of \\ Anesthesiology, The Central Hospital of Enshi Autonomous Prefecture, Enshi, Hubei 445000, P.R. China
}

Received December 8, 2017; Accepted September 13, 2018

DOI: $10.3892 /$ etm.2018.7022

\begin{abstract}
Electroconvulsive therapy (ECT) is an efficient therapy for major depression and modern ECT requires anesthesia to enhance safety. However, the commonly used anesthetic, propofol, may weaken the treatment efficacy. A recent study confirmed that ketamine rapidly reduced the symptoms of depression in affected patients. A previous study found that electroconvulsive seizure (ECS), the animal model for ECT, under anesthesia of low-dose ketamine combined with propofol could enhance the antidepressant efficacy and improve the cognitive performance. The present study aimed to investigate the responses to different charges $(0,60$, 120,180 or $240 \mathrm{mC}$ ) of ECS under compound anesthetics, ketamine combined with propofol, in stressed rats and the underlying mechanisms to aid in optimization of treatment regimens. The results indicated that ECS exhibited an improved antidepressant effects at $120 \mathrm{mC}$ compared with $60 \mathrm{mC}$, however, no significant differences in antidepressant effects were identified among the 120, 180 and $240 \mathrm{mC}$ groups. Furthermore, rats subjected to ECS at $120 \mathrm{mC}$ exhibited the best cognitive performance. The phosphorylation levels of calcium/calmodulin-dependent protein kinase II $\alpha$ (CaMKII $\alpha$ ) at Thr286, glutamate receptor 1 (GluR1) at Ser831 and cAMP-response element-binding protein (CREB) at the Ser133 were higher in the $120-\mathrm{mC}$ group compared with all other groups. These results indicated that the ECS at medium intensity $(120 \mathrm{mC})$ with administration of compound anesthetics may exert an improved therapeutic effect on depression compared with other intensities $(0,60,180$ and $240 \mathrm{mC})$. The
\end{abstract}

Correspondence to: Dr Xianlin Zhu, Department of Anesthesiology, The Central Hospital of Enshi Autonomous Prefecture, 158 Wuyang Road, Enshi, Hubei 445000, P.R. China E-mail: zhuxianlin8@sohu.com

Key words: calcium/calmodulin-dependent protein kinase II, electroconvulsive seizure, glutamate receptor 1, ketamine, protein kinase A results also suggested that the improvement in cognitive function in stressed rats may be attributed to the phosphorylation of CaMKII $\alpha$ (Thr286), GluR1 (Ser831) and CREB (Ser133).

\section{Introduction}

Depression is a prevalent and recurrent psychological illness that results in a significant burden for individuals and the society (1). Previous studies indicated that electroconvulsive therapy (ECT) is an efficient and rapid treatment for major depression, especially for patients with drug-resistant and refractory depression $(2,3)$. Modern ECT requires administration of anesthetic agents and muscle relaxants to avoid the side effects of treatment, including headache, muscle ache and bone fracture (4). At present, propofol is the most frequently used general anesthetic during ECT (5). Previous studies indicated that propofol could alleviate the cognitive impairment induced by electroconvulsive seizure (ECS) $(6,7)$. However, certain studies suggested that propofol may interfere with the efficacy of ECT due to its anticonvulsant properties $(8,9)$. Therefore, an improved anesthetic regimen for ECT is required.

In the last 40 years ketamine has been administered as an anesthetic to several million people $(10,11)$. Ketamine is considered an anesthetic and a rapid-acting antidepressant $(12,13)$. A clinical study revealed that a single ketamine infusion could rapidly reduce the levels of anhedonia in treatment-resistant major depression (14). Notably, the anesthetic dose of ketamine was positively associated with significant adverse psychological effects, including delusion and hallucination $(15,16)$. These disadvantages may limit the clinical use of ketamine, especially for patients with psychiatric disorders. Based on these concerns, a previous study revealed that compared with propofol anesthesia, a low, subanesthetic dose of ketamine combined with propofol could enhance the antidepressant efficacy and further improve the cognitive performance following ECS, an animal analog of human ECT, in stressed stressed rats (17). As the stimulus intensity is germane to both therapeutic and adverse effects of ECT (18), the appropriate stimulus intensity of this modified ECS (MECS) with the introduction of compound anesthetics, requires further study. Furthermore, the biological processes 
underlying the protective effect of MECS on cognitive function remain to be elucidated.

Previous studies revealed that the glutamatergic transmitter system, including N-methyl-D-aspartic acid (NMDA) and $\alpha$-amino-3-hydroxy-5-methyl-4-isoxazolepropionic acid (AMPA) glutamate receptors, was crucial in the pathological physiology of depression and cognitive function $(19,20)$. It is commonly accepted that ketamine exerts its biological effects by inhibiting the NMDA receptor (14). However, another study suggested that ketamine may exert rapid antidepressant-like effects by enhancing the AMPA receptor relative to NMDA receptor throughput in critical neuronal circuits (21). Furthermore, previous studies indicated that the antidepressant effects induced by ketamine or MECS were associated with glutamate receptor 1 (GluR1) (17,21). The AMPA receptor is made up of heterotetrameric complexes comprising at least two of four subunits (GluR1-4) (22). Differences in subunit composition confer specific physiological properties to AMPA receptor function and the phosphorylation of subunits is necessary for maintaining the function of this receptor (23). GluR1 was reported to be involved in the cognitive function $(24,25)$. GluR1 subunit is recruited into the hippocampal synapses to alter the cognitive behavior with the phosphorylation at Ser831 [GluR1 (Ser831)] by calcium/calmodulin-dependent protein kinase II (CaMKII), and at Ser845 [pGluR1 (Ser845)] by protein kinase A (PKA) (24). In addition to the expression of CaMKII and PKA, the activities of these two protein kinases could also affect the phosphorylation of GluR1 (25). Therefore, the current study hypothesized that the improved cognitive performance induced by MECS may be associated with the phosphorylation levels of GluR1 and the expression and activities of these two protein kinases in the hippocampus. CaMKII $\alpha$ is the dominant isoform of CaMKII in the brain (26). The hallmark feature of CaMKII regulation is the generation of autonomous kinase activity by Thr286 autophosphorylation (26). In contrast to CaMKII, the activity of PKA can be determined by the relative expression of the regulatory subunit to the catalytic subunit (25). The effects of different charges of MECS on PKA- and CaMKII $\alpha$-dependent GluR1 phosphorylation have not been previously reported.

In the present study, five different stimulus intensities of MECS were used to evaluate their efficacies on depression-like and cognitive behaviors in stressed rats, and to investigate the potential roles of the expression of GluR1, phospho (p)GluR1 (Ser831), pGluR1 (Ser845), PKA regulatory subunit (PKR2), PKA catalytic subunit (PKA $\beta$ ), CaMKII $\alpha$, pCaMKII $\alpha$ (Thr286), cAMP-response element-binding protein (CREB) and pCREB (Ser133) in these processes.

\section{Materials and methods}

Animals. A total of 104 healthy adult male Sprague-Dawley rats (weight, 200-250 g; age, 2-3 months) were obtained from the Laboratory Animal Center of Chongqing Medical University (Chongqing, China). Animals were housed under standard laboratory conditions (temperature, $22-24^{\circ} \mathrm{C}$; air humidity, 55-60\%; 12-h light/dark cycle) with food and water available ad libitum. Rats were allowed to acclimatize for 1 week before further experiments. All procedures were approved by the Ethics Committee of Chongqing Medical University in accordance with the animal care guidelines of the National Institutes of Health and Use of Laboratory Animals (27).

Chronic unpredictable mild stress procedure. The rat model of depression was established by chronic unpredictable mild stress (CUMS). This experimental protocol was adopted from previous studies with a minor modification (28-30). Briefly, the rats were randomly administered the following stressors for 28 days: Damp sawdust for $24 \mathrm{~h}$ of continuous lighting, swimming in cold water $\left(4^{\circ} \mathrm{C}\right)$ for $5 \mathrm{~min}$, water deprivation for $24 \mathrm{~h}$, food deprivation for $24 \mathrm{~h}$, shaking for $20 \mathrm{~min}$ (once per sec), tail pinching for $1 \mathrm{~min}$, cage tilting $\left(45^{\circ}\right)$ for $24 \mathrm{~h}$, or social crowding for $24 \mathrm{~h}$. All stressor stimuli were randomly scheduled and repeated within 4 weeks to prevent habituation and increase unpredictability.

Drugs and treatment. Propofol and ketamine were purchased from AstraZeneca (cat no. H20080473; Cambridge, UK) and Jiangsu Hengrui Medicine Co., Ltd. (cat no. H32022820; Lianyungang, China), respectively. Based on previous results, a constant dosage of anesthetics, ketamine $(10 \mathrm{mg} / \mathrm{kg})$ and propofol $(80 \mathrm{mg} / \mathrm{kg})$, was administered intraperitoneally (i.p.) 10 min prior to ECS treatment in each group (17). On the day of the experiment, the rats were anesthetized to prepare them for MECS treatments. Anesthesia time was defined as the time from the loss of righting reflex until the regaining of it. The animals were ventilated with a small animal ventilator (TKR-200C; Jiangxi Teli Anaesthesia \& Respiration Equipment Co., Ltd., Nanchang, China). The mean arterial pressure, heart rate and body temperature were continuously monitored using a multichannel electrophysiological recording system (model, RM6240; Chengdu Instrument Factory, Inc., Chengdu, China). The levels of $\mathrm{PaO}_{2}, \mathrm{PaCO}_{2}$ and the acid-base status were adjusted to ensure hemodynamic stability. The rats were excluded from the experiment if they were hemodynamically unstable (mean arterial pressure $<10.7 \mathrm{kPa}$ ) or if their blood gas levels were not within the normal range $\left[\mathrm{PaO}_{2}\right.$ $>13.6 \mathrm{kPa}$; $\mathrm{pH}$ 7.35-7.45; $\mathrm{PaCO}_{2}$ 4.67-6.00 kPa; base excess of -2-2 $\mathrm{mEq}$ ] (31). In the present study, no rats were excluded due to hemodynamic instability or gas levels.

The experimental design is summarized in Fig. 1. Following the completion of the CUMS procedure, one group of stressed rats was used for measuring seizure threshold in ECS with the compound anesthetics (ST group, $n=8$ ). The other stressed rats were allocated to six groups $(n=96)$ : i) Group D received normal saline solution ( $8 \mathrm{ml} / \mathrm{kg}$, i.p.) once a day; ii) groups M0, M60, M120, M180 and M240 received ECS treatment with stimulus intensities of $0,60,120,180$, and $240 \mathrm{mC}$, respectively, following treatment with the same anesthetic protocol [ketamine $(10 \mathrm{mg} / \mathrm{kg})$ and propofol $(80 \mathrm{mg} / \mathrm{kg})$, i.p.]. ECS was delivered via ear clip electrodes using a Niviqure ECT system (Niviqure Meditech, Pvt., Ltd., Bangalore, India) (32). The following stimulus parameters were used: Bidirectional square wave pulses (amplitude, $0.8 \mathrm{~A}$; width, $1.5 \mathrm{~ms} ; 125 \mathrm{~Hz}$ ) with a duration of $0.4,0.8,1.2$ or $1.6 \mathrm{sec}$ to generate charges of $60,120,180$ and $240 \mathrm{mC}$, respectively. These treatments were administered once daily for 7 days. Oxygen was given to the rats that underwent ECS and their saturation of blood oxygen $\left(\mathrm{SpO}_{2}\right)$ was monitored. Only rats with a value of $\mathrm{SpO}_{2} \geq 95 \%$ were included. 

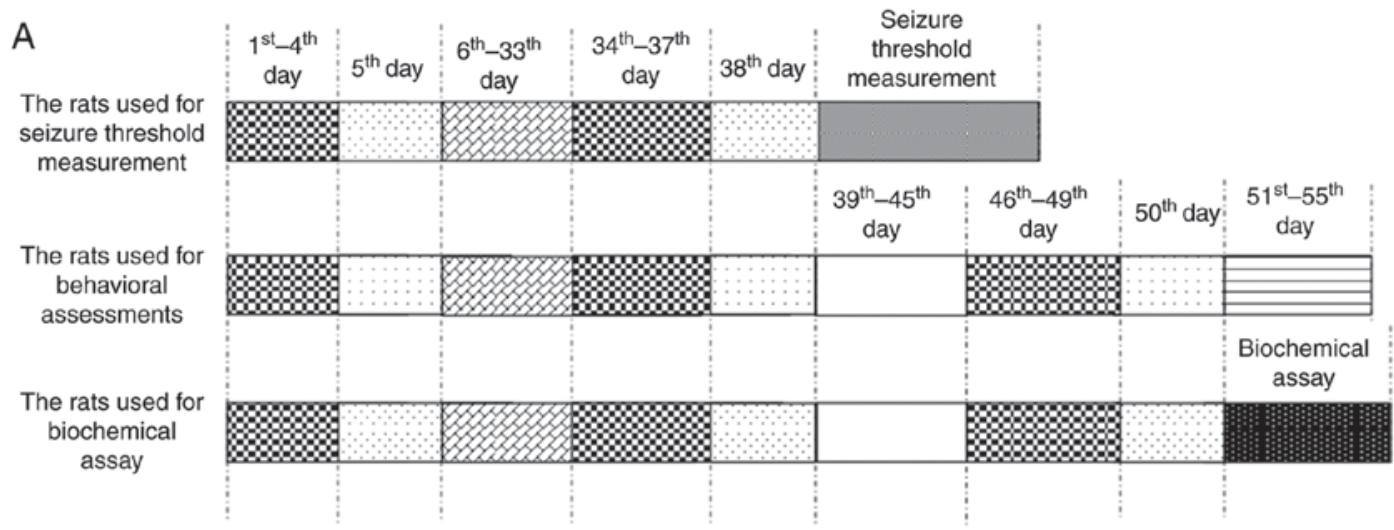

Sucrose preference test Open field test $\quad$ CUMS procedure

Normal saline treatment or different charges of ECS with the compound anesthetics treatment

Morris water maze test

Seizure threshold measurement

Biochemical assay

B

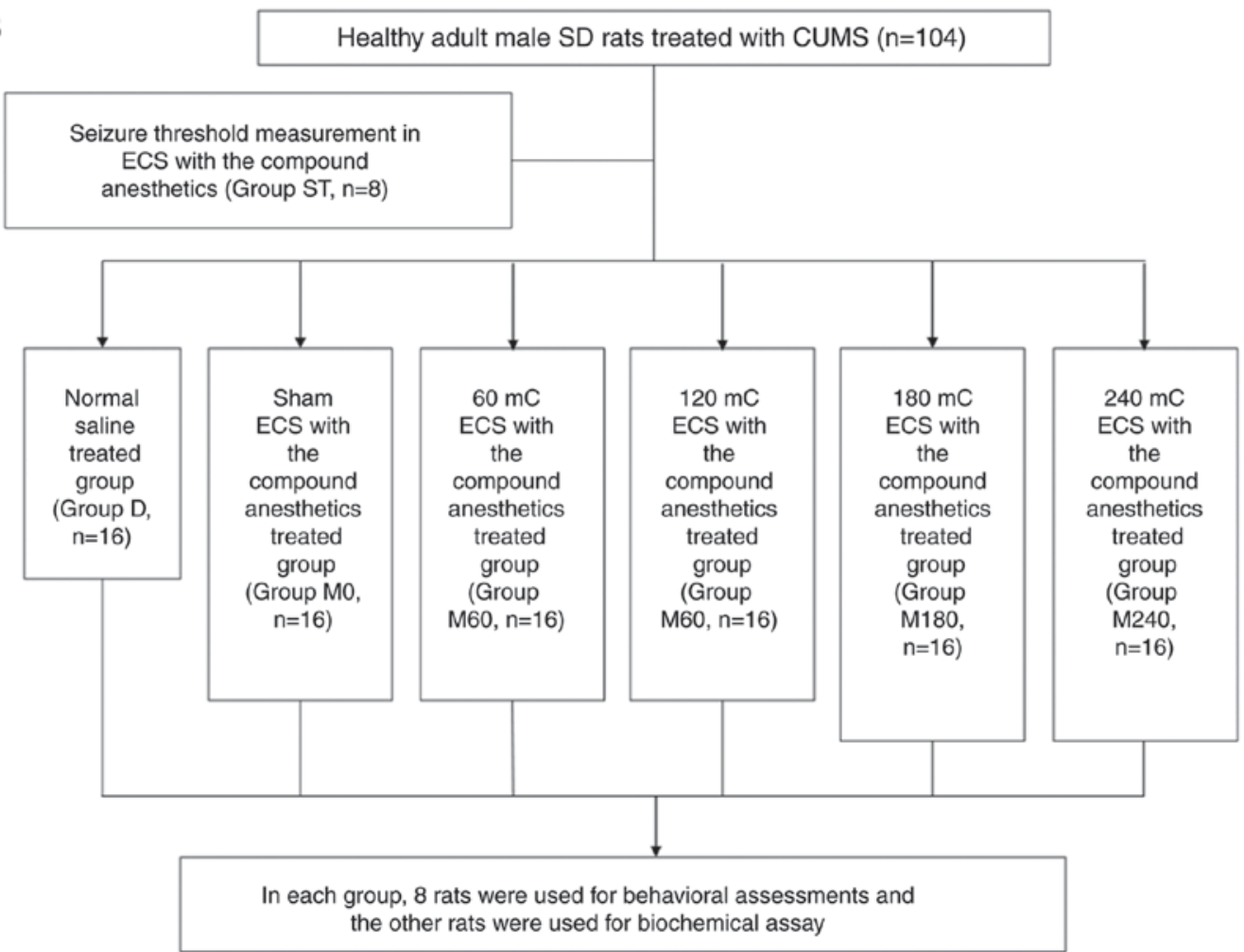

Figure 1. Schematic overview of all group assignments and experimental protocols. (A) Treatment timeline for the use of stressed rats for ECS seizure threshold measurement, behavioral tests and biochemical assays. (B) Group allocation and experimental protocols. SD, Sprague-Dawley; CUMS, chronic unpredictable mild stress; ECS, electroconvulsive seizure; compound anesthetics, ketamine $(10 \mathrm{mg} / \mathrm{kg})$ and propofol $(80 \mathrm{mg} / \mathrm{kg})$ administered via intraperitoneal injection.

A previous study demonstrated that the seizure threshold of rats with propofol anesthesia was $\sim 40 \mathrm{mC}$ (33). Furthermore, a clinical study reported that ketamine may slightly increase the seizure duration induced by ECT (13). Therefore, the current study tested the initial ECS stimuli at $30 \mathrm{mC}$ in each rat of the ST group. If this stimulus did not induce a general tonic seizure, the charge was increased by $1 \mathrm{mC}$ for another ECS on the following day. ECS stimulation was increased by $1 \mathrm{mC}$ at a time until the rat exhibited an obvious tonic seizure. The ECS charge at which a general tonic seizure occurred was recorded as the seizure threshold of the rat.

Sucrose preference test. Anhedonia, an indication of depressive disorder in rats, was defined as a reduction in sucrose preference. Sucrose preference was measured as described in a previous study (33). Following deprivation of water and food 
for $23 \mathrm{~h}$, the rats were given free access to two preweighed bottles for $1 \mathrm{~h}$, one filled with $1 \%$ sucrose solution and the other filled with water. The position of the two bottles was rotated every $30 \mathrm{~min}$ to prevent place preference. The two bottles were subsequently weighed to measure the percentage of the consumed sucrose solution. The sucrose preference percentage (SPP) was calculated according to the following formula: SPP $(\%)=$ sucrose solution consumption $(\mathrm{g})$ x 100/[water consumption $(\mathrm{g})+$ sucrose solution consumption $(\mathrm{g})](7)$.

Open field test (OFT). For the OFT, a spontaneous locomotor activity was measured as described in a previous study (34). Experimental animals were placed at the center of an open box $\left(100 \times 100 \times 50 \mathrm{~cm}^{3}\right)$. The floor was divided into 25 equal squares with the walls painted black. The horizontal locomotor activities (segments crossed with all four paws) and vertical exploratory activities (standing on hind paws) were scored. The number of horizontal activities (crossing) and vertical activities (rearing) performed by each rat was observed for $3 \mathrm{~min}$.

Morris water maze (MWM). To assess the spatial performance function, the rats were tested in a MWM, which was a circular pool (diameter, $150 \mathrm{~cm}$; height, $50 \mathrm{~cm}$ ) with a platform $1-2 \mathrm{~cm}$ below the surface of the warm water $\left(22 \pm 1^{\circ} \mathrm{C}\right)(35)$. The maze was divided into four equal quadrants: SW, NW, SE and NE. Each rat was placed into the water randomly from a quadrant and allowed a maximum of $60 \mathrm{sec}$ to find the submerged platform located at the center of the $\mathrm{NE}$ quadrant. If the rats failed to complete the task within $1 \mathrm{~min}$, they were gently guided to the platform where they remained for $15 \mathrm{sec}$. Each rat was given four trials/day for 5 consecutive days. The swimming speed, swimming distance and escape latency (EL) to find the platform were recorded using a video tracking system device with a data analysis program (Anhui Zhenghua Bio-Tech Co., Ltd., Anhui, China). On day 6, the platform was removed and the probe trial was performed for $60 \mathrm{sec}$. The swim time spent in the platform quadrant was recorded as the space exploration time.

Western blot analysis. Following the spatial memory retention test, eight rats from each group were humanely sacrificed and the bilateral hippocampus was rapidly removed on ice prior to storage at $-80^{\circ} \mathrm{C}$. Tissues were homogenized using the RIPA lysis buffer (Beyotime Institute of Biotechnology, Shanghai, China) and the supernatants were collected after centrifugation at $12,000 \mathrm{x}$ g for $15 \mathrm{~min}$ at $4^{\circ} \mathrm{C}$. After a bovine serum albumin microassay (Pierce BCA Protein Assay Kit; cat. no. 23225; Pierce; Thermo Fisher Scientific, Inc., Waltham, MA, USA) and spectrophotometry to assess the protein levels, total protein was separated by 4-12\% SDS-PAGE and transferred to polyvinylidene fluoride membranes. The membranes were blocked with $5 \%$ nonfat milk for $1 \mathrm{~h}$ at $37^{\circ} \mathrm{C}$ and sub sequently incubated with appropriate primary antibodies overnight at $4^{\circ} \mathrm{C}$ : GluR1 (cat. no. ab31232), pGluR1 (Ser831; cat. no. ab109464), pGluR1 (Ser845; cat. no. ab76321), PKR2 (cat. no. ab38949), PKA $\beta$ (cat. no. ab75993), CREB (cat. no. ab31387), pCREB (Ser133; cat. no. ab32096; all Abcam, Cambridge, UK),

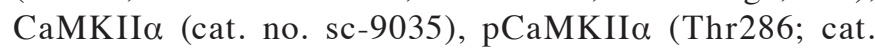
no. sc-12886-R; both Santa Cruz Biotechnology, Inc., Dallas,
TX, USA) and GAPDH (cat. no. AF1186; Beyotime Institute of Biotechnology; all 1:1,000). After washing, the membranes were incubated with biotinylated secondary antibodies (cat. no. A0208; 1:2,000; Beyotime Institute of Biotechnology) for $1 \mathrm{~h}$ at $37^{\circ} \mathrm{C}$. Blots were developed using a chemiluminescent substrate (BeyoECL Plus kit; cat. no. P0018; Beyotime Institute of Biotechnology) and analyzed semi-quantitatively using the Bio-Rad Quantity One software (version 4.4.0; Bio-Rad Laboratories, Inc., Hercules, CA, USA). To determine the relative expression of these proteins, they were normalised to GAPDH.

Immunohistochemistry. The remaining rats in each group $(n=8)$ were anesthetized and transcardially perfused using $4 \%$ paraformaldehyde (PFA) in normal saline. Their brains were removed and stored for at least $24 \mathrm{~h}$ in $4 \%$ PFA at $4^{\circ} \mathrm{C}$ before dehydration and embedding in paraffin. The hippocampi were cut into $10-\mu \mathrm{m}$ thick sections in the coronal plane and mounted on glass slides. The immunoreactivity of pGluR1 (Ser831) and pCaMKII $\alpha$ (Thr 286) was determined as described in a previous study (36). Briefly, sections of the rat brain were incubated overnight at $4^{\circ} \mathrm{C}$ with anti-pGluR1 (Ser831) and anti-pCaMKII $\alpha$ (Thr 286; both 1:100) antibodies, and subsequently incubated for another $1 \mathrm{~h}$ at $37^{\circ} \mathrm{C}$ with biotin-labeled secondary antibodies (1:50; Beyotime Institute of Biotechnology, cat. no. A0208). The primary antibody was visualized using brown 3,3'Diaminobenzidine substrate (Wuhan Boster Biological Technology, Ltd., Wuhan, China). The CA1 region in the hippocampus was observed using a light microscope (Olympus BX60; Olympus Corporation, Tokyo, Japan) at a magnification of $\mathrm{x} 400$ and analyzed using the Image-Pro Plus software (version 6.0; Media Cybernetics, MD, USA).

Statistical analysis. Statistical analysis was performed using SPSS (version 17.0; SPSS Inc., IL, USA). All data are presented as the mean \pm standard deviation. Statistical significance was determined using one-way analysis of variance. The Student-Newman-Keuls test was used to compare differences among the groups. The repeatedly measured data were analyzed using repeated-measures analysis of variance. $\mathrm{P}<0.05$ was considered to indicate a statistically significant difference.

\section{Results}

Seizure threshold. The seizure threshold of CUMS rats treated with compound anesthesia was $41.25 \pm 5.50 \mathrm{mC}$.

Sucrose preference test. The SPP in each CUMS group exhibited a significant decrease compared with the respective group before CUMS (all $\mathrm{P}<0.05$ ), however no significant differences were found among the groups $(\mathrm{F}=0.735, \mathrm{P}=0.601$; Fig. 2A). Following the administration of MECS at different charges, the SPP in groups M60, M120, M180 and M240 significantly increased compared with the respective groups before MECS treatments (all $\mathrm{P}<0.05$ ). Compared with group M60, the SPP increased in group M120 ( $\mathrm{P}<0.05)$. Furthermore, no significant differences were identified in SPP between groups D and M0 $(\mathrm{P}=0.823)$. 

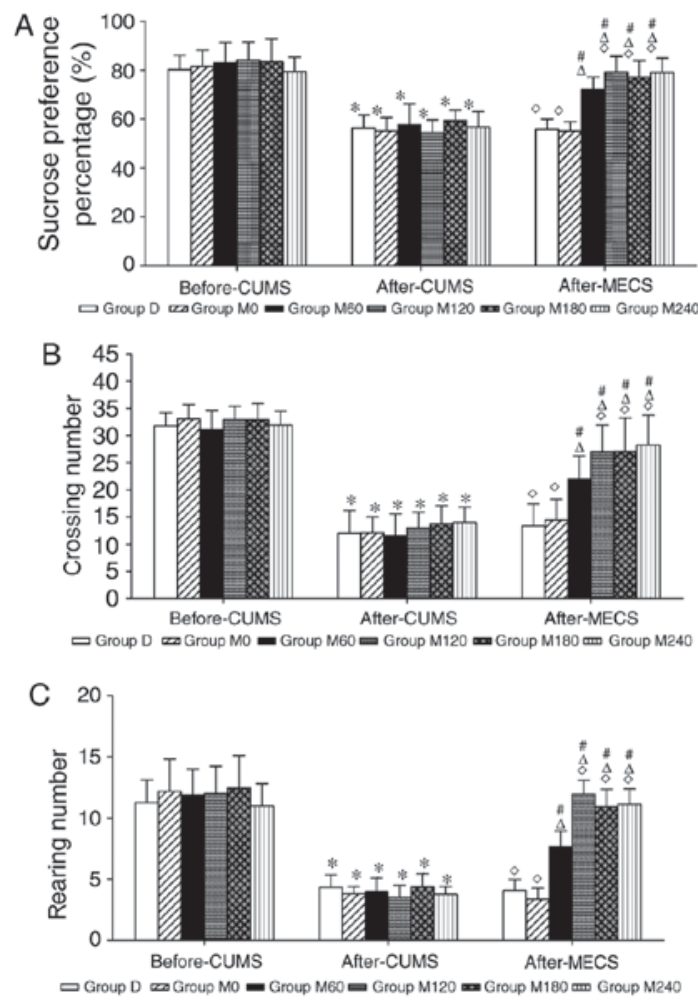

Figure 2. Effects of different charges of MECS on the behavioral performances of animals based on SPP and OFT. (A) SPP. (B) Crossing numbers (C) Rearing numbers. Data are expressed as the mean \pm standard deviation ( $\mathrm{n}=8$ each group). ${ }^{\text {}} \mathrm{P}<0.05$ vs. the respective group before CUMS; ${ }^{*} \mathrm{P}<0.05$ vs. the respective group after CUMS; ${ }^{\triangle} \mathrm{P}<0.05$ vs. group $\mathrm{D}$ after MECS; and ${ }^{\circ} \mathrm{P}<0.05$ vs. M60 group after MECS. MECS, modified electroconvulsive seizure; SPP, sucrose preference percentage; OFT, open field test; CUMS, chronic unpredictable mild stress.

Open field test. Following the CUMS treatment, rats exhibited decreased locomotor activity $(\mathrm{P}<0.05)$ and rearing numbers $(\mathrm{P}<0.05)$ in the OFT compared with the respective groups before the CUMS administration (Fig. 2B and C). No significant differences in OFT scores were found among the CUMS groups (number of crossings, $\mathrm{F}=0.698$ and $\mathrm{P}=0.628$; rearing, $\mathrm{F}=1.038$ and $\mathrm{P}=0.408$ ). Following the MECS treatment at different charges, the number of crossings and rearing in groups M60, M120, M180 and M240 significantly increased compared with the respective groups before the MECS treatment (all $\mathrm{P}<0.05)$. The number of crossings and rearing increased in the M120 group compared with the M60 group (number of crossings, $\mathrm{P}<0.05$; rearing, $\mathrm{P}<0.05$ ). Furthermore, no significant differences were observed in the number of crossings and rearing between groups D and M0 (number of crossings, $\mathrm{P}=0.672$; rearing, $\mathrm{P}=0.235$ ).

Morris water maze. During the MWM test, the swimming speed of CUMS rats exhibited no significant differences among the groups (data not shown). The mean latencies to find the submerged platform decreased progressively during the five consecutive training days for all animals. Following the MECS treatment, the time to find the platform was shorter in the M120 group compared with the other groups $(\mathrm{P}<0.05)$. Furthermore, group M0 exhibited no significant decrease in EL compared with group D each day (P>0.05; Fig. 3A). Similar
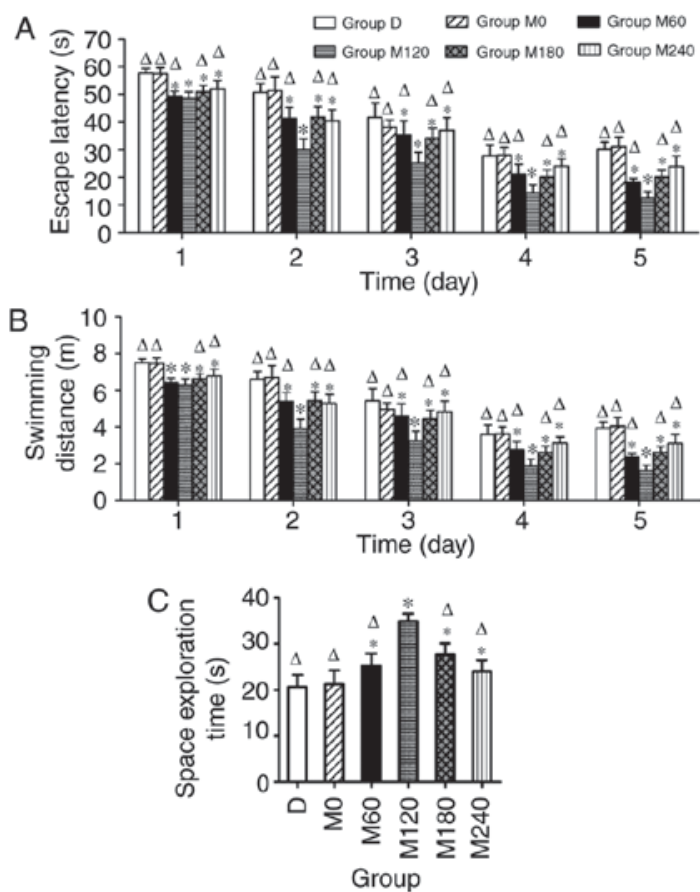

Figure 3. Effects of different charges of modified electroconvulsive seizure on the behavioral performance of animals based on the Morris water maze experiment. (A) Escape latency. (B) Swimming distance. (C) Space exploration time. Data are expressed as the mean \pm standard deviation $(n=8$ each group). ${ }^{*} \mathrm{P}<0.05$ vs. group $\mathrm{D}$ and ${ }^{\Delta} \mathrm{P}<0.05$ vs. group $\mathrm{M} 120$.

results were found for the swimming distance (Fig. 3B). When the platform was removed for spatial memory testing, group M120 exhibited a significantly longer dwelling time in the former platform quadrant. No differences were identified between groups D and M0 (Fig. 3C).

Expression levels of PKA subunits and GluR1, and phosphorylation of GluRl (Ser 845) in the hippocampus. In the present study, PKAR2, PKA $\beta$ (Fig. 4A), GluR1 and pGluR1 (Ser845; Fig. 4B) were assessed via western blotting. No significant differences were identified in the expression of PKAR2 $(\mathrm{F}=0.448$ and $\mathrm{P}=0.810)$ and $\mathrm{PKA} \beta(\mathrm{F}=0.803$ and $\mathrm{P}=0.562)$ subunits of PKA (Fig. 4C). Furthermore, analysis of variance did not reveal any statistically significant effects of MECS on the expression of GluR1 $(\mathrm{F}=1.059$ and $\mathrm{P}=0.415)$ and $\mathrm{pGluR} 1$ (Ser 845; $F=0.365$ and $P=0.866$ ) in the hippocampus (Fig. 4D).

CaMKII $\alpha$ activity and GluRl (Ser831) phosphorylation in the hippocampus. In the present study, CaMKII $\alpha$, pCaMKII $\alpha$ (Thr286; Fig. 5A) and pGluR1 (Ser831; Fig. 5B) were assessed by western blotting. As presented in Fig. 5B, anesthesia combined with ECS treatments did not induce significant effects on the hippocampal expression of CaMKII $\alpha(\mathrm{F}=0.462$ and $\mathrm{P}=0.799)$, however the treatments altered the pCaMKII $\alpha$ (Thr 286) levels $(\mathrm{F}=34.597$ and $\mathrm{P}<0.01)$. Additionally, compared with the other five groups, pCaMKII $\alpha$ (Thr 286) expression significantly increased in the M120 group $(\mathrm{P}<0.01)$, while the expression of this protein was lower in groups D and M0 compared with all other groups (all $\mathrm{P}<0.01$ ). The expression of pCaMKII $\alpha$ (Thr286) and pGluR1 (Ser831) were also examined via immunohistochemistry (Fig. 5C). The expression of pGluR1 (Ser831) 
A

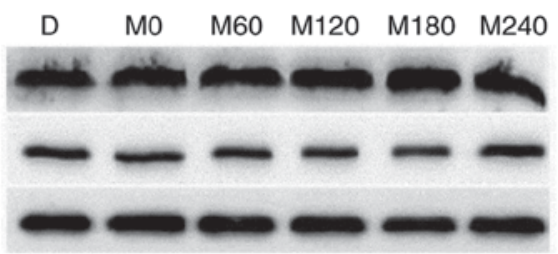

PKAB

PKR2

GAPDH
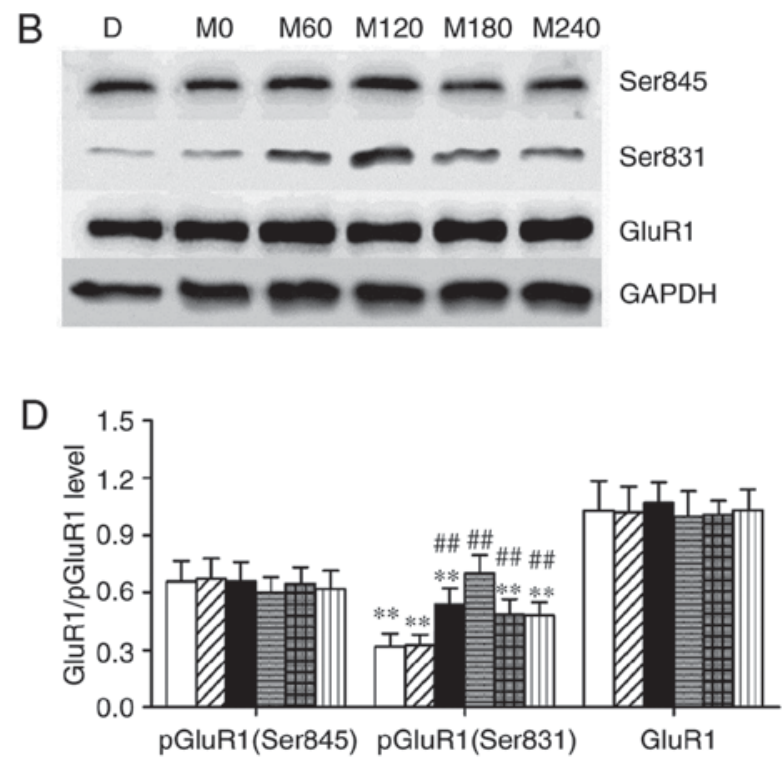

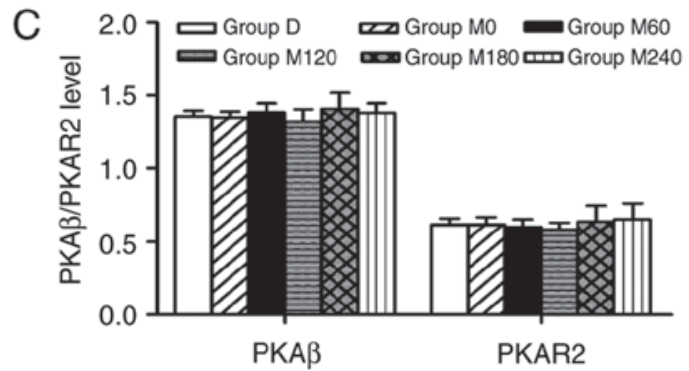

Figure 4. Effects of different charges of modified electroconvulsive seizure on the expression levels of PKA, GluR1 and the phosphorylated forms of GluR1 in the hippocampus. (A) Western blot analyses were conducted to examine the expression levels of PKA $\beta$ and PKR2 in hippocampal tissue. (B) Western blot analyses were conducted to examine the expression levels of pGluR1 (Ser845), pGluR1 (Ser831) and GluR1 in hippocampal tissue. (C) Quantification of PKA $\beta$ and PKR2 expression relative to GAPDH. (D) Quantification of pGluR1 (Ser845), pGluR1 (Ser831) and GluR1 expression relative to GAPDH. Data are presented as the mean \pm standard deviation (biochemical assay, $n=8$ in each group). ${ }^{\# \#} \mathrm{P}<0.01$ vs. group $\mathrm{D}$ and ${ }^{* *} \mathrm{P}<0.01$ vs. group $\mathrm{M} 120$. GluR1, glutamate receptor 1; p, phosphorylated; PKA, protein kinase A; PKR2, PKA regulatory subunit; PKA $\beta$, PKA catalytic subunit.

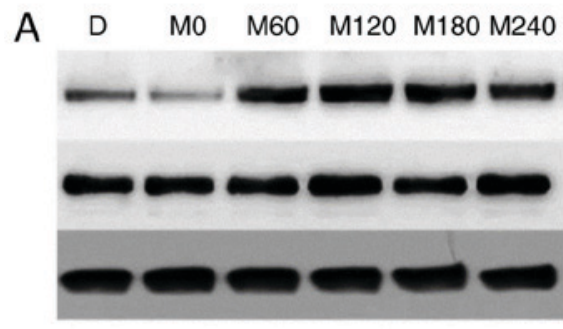

Thr 286

CaMKIl $\alpha$

GAPDH

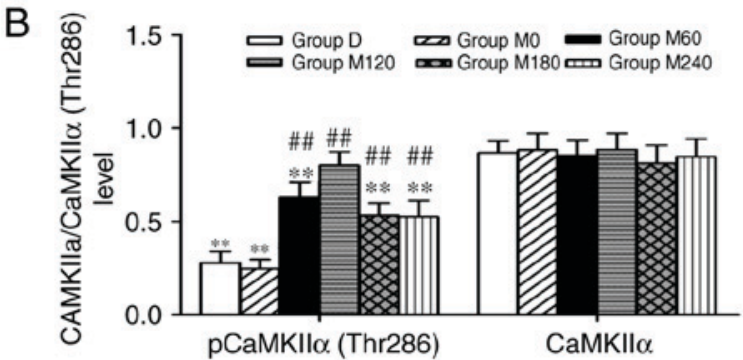

M120
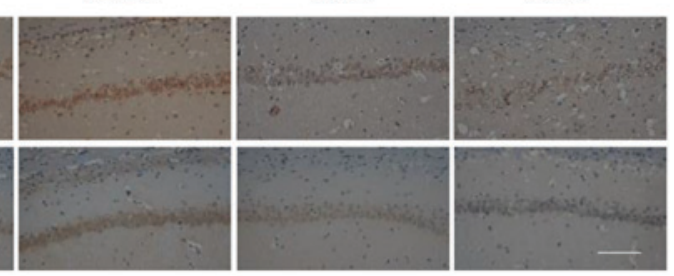

Thr286

Se831

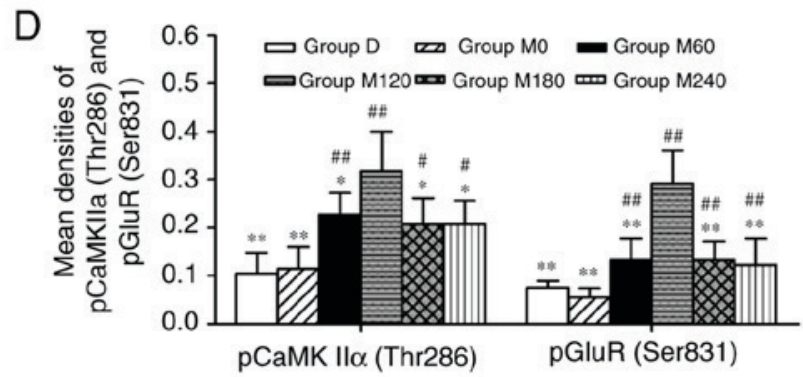

Figure 5. Effects of different charges of modified electroconvulsive seizure on the levels of CaMKII $\alpha$ and pCaMKII $\alpha$ (Thr286) in the hippocampus. (A) Western blot analyses were conducted to examine the expression levels of CaMKII $\alpha$ and pCaMKII $\alpha$ (Thr286) expression in hippocampal tissue. (B) Relative expression of CaMKII $\alpha$ and pCaMKII $\alpha$ (Thr286). (C) Representative images of pGluR (Ser831) and pCaMKII $\alpha$ (Thr286) expression in the CA1 region of the hippocampus (magnification, x400). (D) Quantitative analysis of pGluR (Ser831) and pCaMKII $\alpha$ (Thr286) expression levels in the hippocampus. Data are presented as the mean \pm standard deviation (biochemical assay, $\mathrm{n}=8$ in each group). ${ }^{\prime \prime} \mathrm{P}<0.05$ and ${ }^{\# \#} \mathrm{P}<0.01$ vs. group $\mathrm{D} ;{ }^{*} \mathrm{P}<0.05$ and ${ }^{* *} \mathrm{P}<0.01$ vs. group $\mathrm{M} 120$. CaMKII $\alpha$, calcium/calmodulin-dependent protein kinase Ii ; p, phosphorylated; GluR1, glutamate receptor 1. 

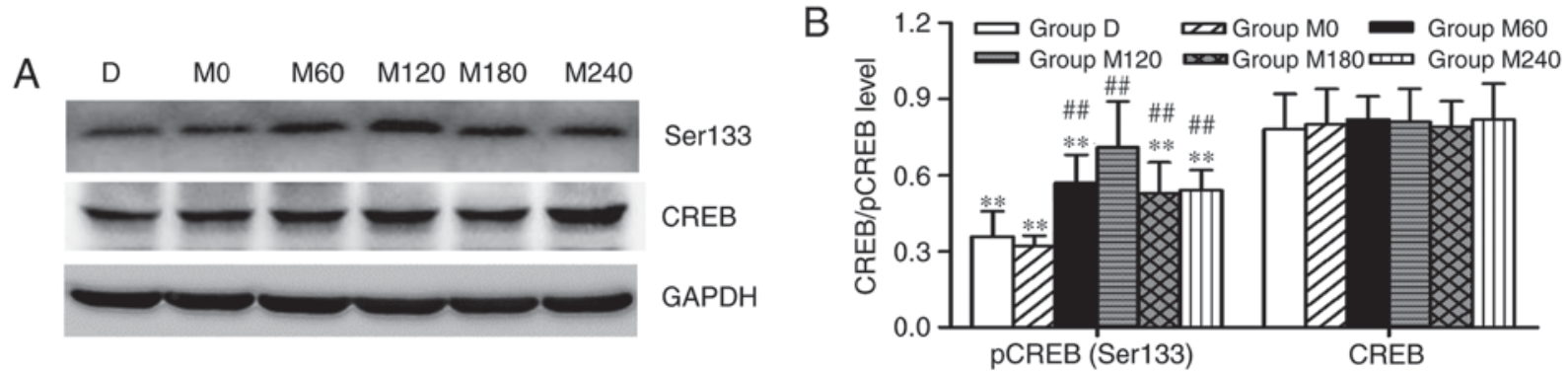

Figure 6. Effects of different charges of modified electroconvulsive seizure on the expression levels of CREB and pCREB (Ser133) in the hippocampus. (A) Western blot analyses were conducted to examine the expression levels of CREB and pCREB (Ser133) in hippocampal tissue. (B) Relative expression of

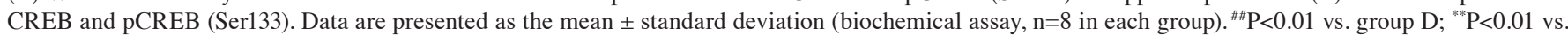
group M120. CREB, cAMP-response element-binding protein; p, phosphorylated.

was the highest in group M120 compared with all other groups (all $\mathrm{P}<0.01$; Fig. 4D). The results of immunohistochemistry were similar to those of western blotting, and indicated that the expression levels of pCaMKII $\alpha$ (Thr 286) and pGluR1 (Ser831) in the CA1 region of the hippocampus increased in group M120 compared with other groups $(\mathrm{P}<0.05$ and $\mathrm{P}<0.01$; Fig. 5D).

Expression levels of CREB and $p C R E B$ (Ser 133) in the hippocampus. The western blot analysis was used to analyze and quantify the protein expression of CREB and pCREB (Ser133) in the hippocampus. No significant alterations in the expression levels of CREB were detected $(\mathrm{F}=1.036$ and $\mathrm{P}=0.427$ ). However, significant differences were identified in the expression levels of pCREB (Ser133) among the six groups $(\mathrm{F}=13.156$ and $\mathrm{P}<0.01)$. Group M120 exhibited a significantly increased level of pCREB (Ser133) in the hippocampus $(\mathrm{P}<0.01)$ compared with the other five groups. Additionally, the expression levels of pCREB were lower in groups D and M0 compared with other groups (all $\mathrm{P}<0.01$; Fig. $6 \mathrm{~A}$ and $\mathrm{B}$ ).

\section{Discussion}

The current study primarily demonstrated that different charges of MECS affected the behavioral performance and protein expression levels in stressed rats. Notably, the medium stimulus intensity $(120 \mathrm{mC})$ of MECS was optimal for improving the antidepressant efficacy and cognitive performance in rats with depression. Furthermore, the underlying cognitive protective mechanisms of the stimulus intensity were associated with the upregulation of pCaMKII $\alpha$ (Thr286), pGluR1 (Ser831) and pCREB (Ser133).

The CUMS procedure, which has been successfully used in previous studies to establish animal models of depression $(17,29,37)$, was first successfully established by Willner et al (38) in 1987. However, it should be noted that CUMS procedures may differ slightly in certain aspects between different laboratories, primarily due to convenience and logistics $(29,39)$. While a certain extent of standardization of the procedure may be desirable, there are no standard factors that distinguish the laboratories in which the procedure operates reliably from those where it does not (39). Anhedonia is one of the primary symptoms of depression, which can be inferred from a reduction in the intake of sweet liquid food $(1,40)$. As expected, the SPP of rats decreased significantly following the
CUMS procedure in the present study, which was consistent with results of a previous study (7). In addition, the CUMS model could also be used to simulate human bradykinesia, which is one of the core symptoms of depression $(41,42)$. OFT is widely used to evaluate the locomotor and exploratory behavior in experimental animals, a reduction in which is a sign of depression $(34,37)$. The stressed rats in the present study exhibited decreased crossing and rearing numbers, indicating apathy and reduced exploration. These results indicated that the depression-like behaviors were successfully modeled using the CUMS procedure. Furthermore, following administration of different charges of MECS, the SPP and OFT performances differed between groups treated with different charges. In addition, the SPP and OFT performances remained unaltered following an increase in charges from medium to high $(\geq 20 \mathrm{mC})$. These results indicated that the ceiling effect of the antidepressant therapy using ECS with the compound anesthetics was reached at the $120 \mathrm{mC}$ charge. One possible explanation for the lack of improvement in antidepressant efficacy following administration of higher stimulus intensities (180 and $240 \mathrm{mC}$ ) in the present study may be associated with the seizure duration, which was an important determinant of the treatment efficacy. A previous study reported that the ECT seizure duration did not exhibit a linear association with the stimulus intensity, and increased intensities may reduce seizure duration (43). However, further studies are required to clarify the underlying mechanism of this phenomenon, as the present study could not monitor the brain electrical activity of the stressed rats due to the lack of equipment. A previous study indicated that pretreatment with propofol could further improve the antidepressant effect induced by ketamine in the forced swimming test (44). However, no statistically significant improvement was found in the depression-like behaviors in rats administered the compound anesthetics only (group M0). The present study hypothesized that this result may be associated with the anesthetic regimen and animal models (exposed to acute or chronic stressors). Wang et al (44) used a subanesthetic adjuvant dose of propofol $(5,10,15$ and $20 \mathrm{mg} / \mathrm{kg}$, i.p.) combined with ketamine $(15 \mathrm{mg} / \mathrm{kg}$, i.p.) to evaluate their safety and efficacy in rats with acute depression-like behavior. In the current study, a low dose of ketamine (10 mg/kg, i.p.) combined with propofol $(80 \mathrm{mg} / \mathrm{kg}$, i.p.), which was equivalent to the clinical dose in humans, was selected as the anesthetics during ECS in chronically stressed rats. Furthermore, Wang et al (44) 
found that the subanesthetic adjuvant dose of propofol with ketamine could not increase the crossing and rearing of the rats compared with the control group. Therefore, combined with clinical-dose propofol, a lower-dose ketamine may not be able to exert a direct antidepressant effect in the chronic stress model of depression. To the best of the authors' knowledge, the anesthetic regimen used in the present study has not been previously reported. However, according to previous studies and relevant preliminary experiments, propofol $(100 \mathrm{mg} / \mathrm{kg}$, i.p.) or ketamine $(50-100 \mathrm{mg} / \mathrm{kg}$, i.p.) could be safely used as general anesthetics for rats (45-47). To minimize the possible adverse effects of ketamine including delusions and hallucinations, a lower dose (10 mg/kg, i.p.) was selected in the present study. Using the transformation formula for ketamine between rats and human patients, the dosage used in the present study was equivalent to the low-dose ketamine commonly used in clinical research for the induction of antidepressant effects in patients (17). A previous study has indicated that administration of $10 \mathrm{mg} / \mathrm{kg}$, i.p. ketamine could prevent stress-evoked dysfunctions in rats (48). Due to the administration of compound anaesthetics, $80 \mathrm{mg} / \mathrm{kg}$, i.p. propofol was used in the current study. This dose has been previously demonstrated to be safe and effective (47). No animal mortality was observed, and, therefore, the compound dosage used in the current study may be appropriate for MECS.

The therapeutic efficacy of ECT is associated with the degree to which the stimulus intensity exceeds the seizure threshold (i.e., the relative intensity) (49). The initial seizure threshold of the stressed rats treated with the compound anesthetic was $\sim 41 \mathrm{mC}$. The relative intensities of 60,120 , 180 and $240 \mathrm{mC}$ used in the present study were $\sim 1.5-, 3-$, 4.5- and 6-fold the seizure threshold, respectively. Although the highest charge was $240 \mathrm{mC}$, all rats survived under the high intensities in the present study, which was in line with the results reported by Luo et al (33). A previous study found that the initial seizure threshold of solo ECS (without the administration of anesthetics) was $10 \mathrm{mC}$ on average (18), and the threshold increased to $40 \mathrm{mC}$ when ECS was administered with propofol (33), suggesting that this anaesthetic may exhibit antiepileptic effects. Based on the result that the initial seizure threshold of ECS co-administered with propofol and low-dose ketamine was $41 \mathrm{mC}$ in the current study, it was hypothesized that low-dose ketamine may not interfere in the seizure effects of ECS.

MWM test is frequently used as to study learning and memory function of animal models $(1,36,50)$. However, the MWM test can lead to epigenetic alterations in the hippocampus and influence the cognitive behavior of animals. Zots et al (51) found that the MWM test caused the formation of both spatial and nonspatial memory. Carter et al (50) demonstrated that MWM training caused distinct epigenetic and gene expression alterations in rat hippocampal neurons (50). Therefore, repeated MWM tests may not accurately reflect the alteration of learning and memory function induced by ECS and anesthetics. Based on these concerns, the current study used 8 rats from each group for the behavioural tests, including the MWM test, and 8 rats from each group for the biochemical assays. The MWM test test was performed only once to prevent changes to the epigenome. This protocol prevented the behavioral tests negatively affecting memory, which can be associated with differential protein expression in the brain of the stressed rats (50). Preliminary studies indicated that the combination of ketamine and propofol exhibited a better cognitive performance compared with the use of propofol alone in the ECS procedure $(17,52)$. The present study further identified an appropriate stimulus intensity of ECS to be applied in combination with anesthetics to improve the cognitive function of stressed rats. The results of the present study confirmed that the medium charge of 120 $\mathrm{mC}$ was the optimal stimulus intensity for the improvement in cognitive function. Receiving the compound anesthetics alone could not improve the cognitive performance and depression-like behaviors in stressed rats. The results of the current study indicated that low-dose ketamine combined with propofol was an appropriate anesthetic protocol for ECS, and $120 \mathrm{mC}$ was the optimal charge under this novel protocol.

A previous study suggested that ketamine, an NMDA receptor antagonist, when administered at a low dose, may induce rapid antidepressant effects (14). Furthermore, low-dose ketamine could ameliorate the cognitive impairment induced by ECS (17). Previous studies suggested that the NMDA receptor is one of the key factors regulating the cognitive function. One study indicated that chronic exposure to arsenic could reduce the learning and memory function accompanied by downregulated levels of NMDA receptor subunit 2 (NR2) and memory-associated proteins proto-oncogene c-Fos and c-Jun (53). However, Maeng et al (21) revealed that both nonselective NMDA antagonists and NR2B-selective antagonists exerted antidepressant effects by enhancing the activity of AMPA receptors relative to NMDA receptors. Furthermore, Chen et al (17) found that low-dose ketamine combined with propofol could improve the cognitive impairment induced by ECS, which may be attributed to modulating the expression of pGluR1. GluR1 receptor subunit is expressed in the majority of brain regions, predominantly in the hippocampus (54). Previous studies suggested that the phosphorylation of GluR1 was necessary for synaptic expression, channel properties of AMPA receptors, synaptic plasticity, spatial learning and memory function $(24,54,55)$. GluR1 Ser831/Ser845 double phosphomutant mice exhibited a significant cognitive deficit (56). Therefore, the GluR1 phosphorylation states modulated by CaMKII and PKA may serve roles in cognitive function. The current study indicated that the expression level of pCaMKII $\alpha$ (Thr286) in group M120 was the highest among the study groups, and was coincident with the cognitive performance in the MWM test. However, no significant alterations in total CaMkII $\alpha$ protein levels were observed. Therefore, these results suggested that the effects of different charges of MECS may be associated with alterations in CaMkII $\alpha$ activity. The results of the present study are consistent with the results of a previous study where an increase in CaMkII activity was associated with the induction of long-term potentiation (LTP) as a model of synaptic plasticity (57). Activation of CaMkII resulted in an increased expression of AMPA receptors, which caused enhanced function of AMPA and was necessary for the induction of LTP (57). Previous studies indicated that PKA acted in parallel with CaMKII in controlling synaptic incorporation of GluR1 $(24,58)$. Therefore, the activation of PKA may also 
affect activity-dependent AMPA receptor trafficking $(24,58)$. The current study identified no marked alterations in the expression levels of the catalytic or regulatory subunits of PKA, suggesting that the alteration in cognitive function among the rats may not be associated with the PKA activity. However, it has been previously demonstrated that the activation of either PKA or CaMKII could modulate the GluR1 phosphorylation (24). One possible explanation for the above results was that repeated ECS, similar to single ECS, exerted its clinical effect primarily through the modulation of AMPA activity mediated by the GluR1 phosphorylation at Ser831 (59). The biochemical results of the present study indicated that GluR1 was significantly phosphorylated at Ser831 following MECS treatment, whereas the phosphorylation of GluR1 at Ser845 was unaltered. Therefore, MECS improved the cognitive performance in stressed rats, which may be due to the activation of the CaMKII-mediated phosphorylation of GluR1 in the hippocampus.

CREB is a key modulator of development, plasticity and neuroprotection in the central nervous system. Alterations in CREB-mediated transcription have been implicated in multiple cognitive and psychiatric disorders including depression and cognitive decline (60). It has been previously demonstrated that CREB activity decreased in the brains of depressed patients, was upregulated following administration of a number of antidepressants, and induced antidepressant effects in rodent hippocampi (61). Qiu et al (62) indicated that CREB could be phosphorylated in response to the activation of GluRs, leading to alterations in synaptic plasticity and neuronal structure. Therefore, the current study detected the expression of CREB and pCREB and found that the medium charge of $120 \mathrm{mC}$ administered with the novel anesthetic protocol markedly upregulated the level of pCREB and did not alter the expression of CREB in the experimental animals. These results further confirmed that the combination of ketamine, propofol and ECS affected the cognitive function of depressed animals associated with synaptic plasticity. The present study suggested that optimal neuroprotective effects require administration of an appropriate stimulus intensity for the improvement of antidepressant activity.

In conclusion, the present study provided evidence that the medium stimulus of $120 \mathrm{mC}$ was the optimal intensity for the treatment of stressed rats using this novel anesthetic protocol. The improved cognitive function was associated with CaMKII-mediated phosphorylation of GluR1 in the hippocampus.

\section{Acknowledgements}

The authors would like to thank Dr Jie Luo (Department of Anesthesiology, The First Affiliated Hospital of Chongqing Medical University, Chongqing, China) for her help in correcting the manuscript.

\section{Funding}

The present study was supported by the National Natural Science Foundation of China (grant no. 81760257), the Science and Technology Department of Sichuan Province (grant no. 2018JY0351) and the Science and the Science and Technology Department of Hubei Province (grant no. 2016CFB368).

\section{Availability of data and materials}

The datasets used and/or analyzed during the present study are available from the corresponding author on reasonable request.

\section{Authors' contributions}

FZ was performed the experiments, data analysis and manuscript preparation. $\mathrm{GH}$ and $\mathrm{XZ}$ wrote and revised the manuscript, and produced the experimental design.

\section{Ethics approval and consent to participate}

The experiment protocols were approved by the Ethical Committee of Chongqing Medical University (reference no. 2017-004).

\section{Patient consent for publication}

Not applicable.

\section{Competing interests}

The authors declare that there is no conflict of interests regarding the publication of this paper.

\section{References}

1. Han J, Wang LU, Bian H, Zhou X and Ruan C: Effects of paroxetine on spatial memory function and protein kinase $\mathrm{C}$ expression in a rat model of depression. Exp Ther Med 10: 1489-1492, 2015.

2. Pittenger Cand Duman RS: Stress, depression, and neuroplasticity: A convergence of mechanisms. Neuropsychopharmacology 33: 88-109, 2008

3. Pagnin D, de Queiroz V, Pini S and Cassano GB: Efficacy of ECT in depression: A meta-analytic review. J ECT 20: 13-20, 2004.

4. Lisanby SH: Electroconvulsive therapy for depression. N Engl J Med 357: 1939-1945, 2007.

5. Patel AS, Gorst-Unsworth C, Venn RM, Kelley K and Jacob Y: Anesthesia and electroconvulsive therapy: A retrospective study comparing etomidate and propofol. J ECT 22: 179-183, 2006.

6. Luo J, Min S, Wei K, Cao J, Wang B, Li P, Dong J and Liu Y: Propofol prevents electroconvulsive-shock-induced memory impairment through regulation of hippocampal synaptic plasticity in a rat model of depression. Neuropsychiatr Dis Treat 10: 1847-1859, 2014.

7. Zhu X, Hao X, Luo J, Min S, Xie F and Zhang F: Propofol inhibits inflammatory cytokine-mediated glutamate uptake dysfunction to alleviate learning/memory impairment in depressed rats undergoing electroconvulsive shock. Brain Res 1595: 101-109, 2015.

8. Rampton A, Griffin R, Durcan J and Stuart C: Propofol and electroconvulsive therapy. Lancet 331: 296-297, 1988.

9. Warnell RL, Swartz CM and Thomson A: Propofol interruption of ECT seizure to reduce side-effects: A pilot study. Psychiatry Res 175: 184-185, 2010.

10. Naughton M, Clarke G, O'Leary OF, Cryan JF and Dinan TG: A review of ketamine in affective disorders: Current evidence of clinical efficacy, limitations of use and pre-clinical evidence on proposed mechanisms of action. J Affect Disord 156: 24-35, 2014.

11. White PF, Way WL and Trevor AJ: Ketamine-its pharmacology and therapeutic uses. Anesthesiology 56: 119-136, 1982.

12. Kranaster L, Kammerer-Ciernioch J, Hoyer C and Sartorius A: Clinically favourable effects of ketamine as an anaesthetic for electroconvulsive therapy: A retrospective study. Eur Arch Psychiatry Clin Neurosci 261: 575-582, 2011. 
13. Okamoto N, Nakai T, Sakamoto K, Nagafusa Y, Higuchi T and Nishikawa T: Rapid antidepressant effect of ketamine anesthesia during electroconvulsive therapy of treatment-resistant depression: Comparing ketamine and propofol anesthesia. J ECT 26: 223-227, 2010.

14. Zarate CA, Singh JB, Carlson PJ, Brutsche NE, Ameli R, Luckenbaugh DA, Charney DS and Manji HK: A randomized trial of an N-methyl-D-aspartate antagonist in treatment-resistant major depression. Arch Gen Psychiatry 63: 856-864, 2006.

15. Corlett PR, Honey GD, Aitken MR, Dickinson A, Shanks DR, Absalom AR, Lee M, Pomarol-Clotet E, Murray GK, McKenna PJ, et al: Frontal responses during learning predict vulnerability to the psychotogenic effects of ketamine: Linking cognition, brain activity and psychosis. Arch Gen Psychiatry 63 611-621, 2006

16. Bowdle AT, Radant AD, Cowley DS, Kharasch ED, Strassman RJ and Roy-Byrne PP: Psychedelic effects of ketamine in healthy volunteers relationship to steady-state plasma concentrations. J Am Soc Anesthesiologists 88: 82-88, 1998.

17. Chen J, Peng LH, Luo J, Liu L, Lv F, Li P, Ao L, Hao XC and Min S: Effects of low-dose ketamine combined with propofol on phosphorylation of AMPA receptor GluR1 subunit and GABAA receptor in hippocampus of stressed rats receiving electroconvulsive shock. J ECT 31: 50-56, 2015.

18. Andrade C, Kurinji S, Sudha S and Chandra JS: Effects of pulse amplitude, pulse frequency and stimulus duration on seizure threshold: A laboratory investigation. J ECT 18: 144-148, 2002.

19. Xu Y, Li X, Wang X, Yao J and Zhuang S: miR-34a deficiency in APP/PS1 mice promotes cognitive function by increasing synaptic plasticity via AMPA and NMDA receptors. Neurosci Lett 670: 94-104, 2018.

20. Treccani G, Kristian GDJ, Wegener G and Müller HK: Differential expression of postsynaptic NMDA and AMPA receptor subunits in the hippocampus and prefrontal cortex of the flinders sensitive line rat model of depression. Synapse 70: 471-474, 2016.

21. Maeng S, Zarate CA Jr, Du J, Schloesser RJ, McCammon J, Chen G and Manji HK: Cellular mechanisms underlying the antidepressant effects of ketamine: role of alpha-amino-3-hydroxy-5-methylisoxazole-4-propionic acid receptors. Biol Psychiatry 63: 349-352, 2008.

22. Greger IH, Ziff EB and Penn AC: Molecular determinants of AMPA receptor subunit assembly. Trends Neurosci 30: 407-416, 2007.

23. Nordgren M, Karlsson T, Svensson M, Koczy J, Josephson A, Olson L, Tingström A and Brené S: Orchestrated regulation of Nogo receptors, LOTUS, AMPA receptors and BDNF in an ECT model suggests opening and closure of a window of synaptic plasticity. PLoS One 8: e78778, 2013.

24. Keifer J and Zheng Z: AMPA receptor trafficking and learning. Eur J Neurosci 32: 269-277, 2010.

25. Bracey JM, Kurz JE, Low B and Churn SB: Prolonged seizure activity leads to increased protein kinase A activation in the rat pilocarpine model of status epilepticus. Brain Res 1283: 167-176, 2009.

26. Coultrap SJ, Zaegel V and Bayer KU: CaMKII isoforms differ in their specific requirements for regulation by nitric oxide. FEBS Lett 588: 4672-4676, 2014.

27. Ren L, Zhang F, Min S, Hao X, Qin P and Zhu X: Propofol ameliorates electroconvulsive shock-induced learning and memory impairment by regulation of synaptic metaplasticity via autophosphorylation of CaMKIIa at Thr 305 in stressed rats. Psychiatry Res 240: 123-130, 2016.

28. Banasr M, Valentine GW, Li XY, Gourley SL, Taylor JR and Duman RS: Chronic unpredictable stress decreases cell proliferation in the cerebral cortex of the adult rat. Biol Psychiatry 62: 496-504, 2007.

29. Karolina P, Barbara B and Grazyna B: Utility of the chronic unpredictable mild stress model in research on new antidepressants. Curr Issue Pharm Med Sci 27: 97-101, 2014.

30. Luo KR, Hong CJ, Liou YJ, Hou SJ, Huang YH and Tsai SJ: Differential regulation of neurotrophin S100B and BDNF in two rat models of depression. Prog Neuropsychopharmacol Biol Psychiatry 34: 1433-1439, 2010.

31. Xie F, Min S, Liu L, Peng L, Hao X and Zhu X: Advanced age enhances the sepsis-induced up-regulation of the $\gamma$ - and a7-nicotinic acetylcholine receptors in different parts of the skeletal muscles. Arch Gerontology Geriatr 65: 1-8, 2016.

32. Segi-Nishida E, Warner-Schmidt JL and Duman RS: Electroconvulsive seizure and VEGF increase the proliferation of neural stem-like cells in rat hippocampus. Proc Natl Acad Sci USA 105: 11352-11357, 2008
33. Luo J, Min S, Wei K, Zhang J and Liu Y: Propofol interacts with stimulus intensities of electroconvulsive shock to regulate behavior and hippocampal BDNF in a rat model of depression. Psychiatry Res 198: 300-306, 2012.

34. Ding L, Zhang X, Guo H, Yuan J, Li S, Hu W, Golden T and Wu N: The functional study of a chinese herbal compounded antidepressant medicine-jie yu chu fan capsule on chronic unpredictable mild stress mouse model. PLoS One 10: e0133405, 2015

35. Cao XZ, Ma H, Wang JK, Liu F, Wu BY, Tian AY, Wang LL and Tan WF: Postoperative cognitive deficits and neuroinflammation in the hippocampus triggered by surgical trauma are exacerbated in aged rats. Prog Neuropsychopharmacology Biol Psychiatry 34: $1426-1432,2010$.

36. Liao WT, Xiao XY, Zhu Y and Zhou SP: The effect of celastrol on learning and memory in diabetic rats after sevoflurane inhalation. Arch Med Sci 14: 370-380, 2018.

37. Tianzhu Z, Shihai Y and Juan D: Antidepressant-like effects of cordycepin in a mice model of chronic unpredictable mild stress Evid Based Complement Alternat Med 2014: 438506, 2014.

38. Willner P, Towell A, Sampson D, Sophokleous S and Muscat R: Reduction of sucrose preference by chronic unpredictable mild stress and its restoration by a tricyclic antidepressant. Psychopharmacology (Berl) 93: 358-364, 1987.

39. Willner P: Validity, reliability and utility of the chronic mild stress model of depression: A 10 -year review and evaluation. Psychopharmacology (Berl) 134: 319-329, 1997.

40. Xinxing W, Wei L, Lei W, Rui Z, Baoying J and Lingjia Q: A neuroendocrine mechanism of co-morbidity of depression-like behavior and myocardial injury in rats. PLoS One 9: e88427, 2014.

41. Garcia LS, Comim CM, Valvassori SS, Réus GZ, Stertz L, Kapczinski F, Gavioli EC and Quevedo J: Ketamine treatment reverses behavioral and physiological alterations induced by chronic mild stress in rats. Prog Neuropsychopharmacol Biol Psychiatry 33: 450-455, 2009.

42. Caligiuri MP and Ellwanger J: Motor and cognitive aspects of motor retardation in depression. J Affect Disord 57: 83-93, 2000.

43. Frey R, Heiden A, Scharfetter J, Schreinzer D, Blasbichler T, Tauscher J, Felleiter P and Kasper S: Inverse relation between stimulus intensity and seizure duration: Implications for ECT procedure. J ECT 17: 102-108, 2001.

44. Wang X, Yang Y, Zhou X, Qu Q, Ou C, Liu L and Zhou S: Propofol pretreatment increases antidepressant-like effects induced by acute administration of ketamine in rats receiving forced swimming test. Psychiatry Res 185: 248-253, 2011.

45. Alves HN, da Silva AL, Olsson IA, Orden JM and Antunes LM: Anesthesia with intraperitoneal propofol, medetomidine, and fentanyl in rats. J Am Assoc Lab Anim Sci 49: 454-459, 2010.

46. Taşkara E, Gör A, Kutlu O, Karagüzel E, Topbaş M and Șenel AC: Does propofol prevent testicular ischemia-reperfusion injury due to torsion in the long term? Pediatr Surg Int 27: 1003-1007, 2011.

47. Kushikata T, Sawada M, Niwa H, Kudo T, Kudo M, Tonosaki M and Hirota K: Ketamine and propofol have opposite effects on postanesthetic sleep architecture in rats: relevance to the endogenous sleep-wakefulness substances orexin and melanin-concentrating hormone. J Anesth 30: 437-443, 2016.

48. Nikiforuk A and Popik P: Ketamine prevents stress-induced cognitive inflexibility in rats. Psychoneuroendocrinology 40: 119-122, 2014.

49. Sackeim HA, Devanand DP and Prudic J: Stimulus intensity, seizure threshold and seizure duration: Impact on the efficacy and safety of electroconvulsive therapy. Psychiatr Clin North Am 14: 803-843, 1991

50. Carter SD, Mifsud KR and Reul JM: Distinct epigenetic and gene expression changes in rat hippocampal neurons after Morris water maze training. Front Behav Neurosci 9: 156, 2015.

51. Zots MA, Ivashkina OI, Ivanova AA and Anokhin KV: Formation of spatial and nonspatial memory in different condensed versions of short-term learning in morris water maze. Bull Exp Biol Med 156: 602-604, 2014.

52. Erdogan Kayhan G, Yucel A, Colak YZ, Ozgul U, Yologlu S, Karlidag R and Ersoy MO: Ketofol (mixture of ketamine and propofol) administration in electroconvulsive therapy. Anaesth Intensive Care 40: 305-310, 2012.

53. Wang D, Wang X, Liu X, Jiang L, Yang G, Shi X, Zhang C and Piao F: Inhibition of miR-219 alleviates arsenic-induced learning and memory impairments and synaptic damage through up-regulating CaMKII in the hippocampus. Neurochem Res 43: 948-958, 2018. 
54. MatsuzakiK, MiyazakiK,Sakai S, YawoH,Nakata N,MoriguchiS, Fukunaga K, Yokosuka A, Sashida Y, Mimaki Y, et al: Nobiletin, a citrus flavonoid with neurotrophic action, augments protein kinase A-mediated phosphorylation of the AMPA receptor subunit, GluR1 and the postsynaptic receptor response to glutamate in murine hippocampus. Eur J Pharmacol 578: 194-200, 2008.

55. Lee HK, Barbarosie M, Kameyama K, Bear MF and Huganir RL: Regulation of distinct AMPA receptor phosphorylation sites during bidirectional synaptic plasticity. Nature 405: 955-959, 2000.

56. Lee HK, Takamiya K, Han JS, Man H, Kim CH, Rumbaugh G, Yu S, Ding L, He C, Petralia RS, et al: Phosphorylation of the AMPA receptor GluR1 subunit is required for synaptic plasticity and retention of spatial memory. Cell 112: 631-643, 2003.

57. Singleton MW, Holbert WH II, Lee AT, Bracey JM and Churn SB: Modulation of CaM kinase II activity is coincident with induction of status epilepticus in the rat pilocarpine model. Epilepsia 46: 1389-1400, 2005.

58. Wu LJ, Ren M, Wang H, Kim SS, Cao X and Zhuo M: Neurabin contributes to hippocampal long-term potentiation and contextual fear memory. PLoS One 3: e1407, 2008.
59. Fumagalli F, Pasini M, Sartorius A, Scherer R Racagni G, Riva MA and Gass P: Repeated electroconvulsive shock (ECS) alters the phosphorylation of glutamate receptor subunits in the rat hippocampus. Int J Neuropsychopharmacol 13: 1255-1260, 2010

60. Tanis KQ, Duman RS and Newton SS: CREB binding and activity in brain: Regional specificity and induction by electroconvulsive seizure. Biol Psychiatry 63: 710-720, 2008.

61. Carlezon WA Jr, Duman RS and Nestler EJ: The many faces of CREB. Trends Neurosci 28: 436-445, 2005.

62. Qiu S and Currás-Collazo MC: Histopathological and molecular changes produced by hippocampal microinjection of domoic acid. Neurotoxicol Teratology 28: 354-362, 2006.

This work is licensed under a Creative Commons

Attribution-NonCommercial-NoDerivatives 4.0 International (CC BY-NC-ND 4.0) License. 